\title{
A Case Study of the Collaborative Approaches to Sustain Open Source Business Models
}

\author{
Shane Coughlan \\ Shimane University \\ Nishikawatsu-cho 1060 \\ Matsue City, Shimane Pref. Japan \\ $+81-852-32-6164$ \\ shane@opendawn.com
}

\author{
Tetsuo Noda \\ Shimane University \\ Nishikawatsu-cho 1060 \\ Matsue City, Shimane Pref. Japan \\ +81-852-32-6164 \\ nodat@soc.shimane-u.ac.jp
}

\author{
Terutaka Tansho \\ Shimane University \\ Nishikawatsu-cho 1060 \\ Matsue City, Shimane Pref. Japan \\ +81-852-60-2293 \\ tansho@riko.shimane-u.ac.jp
}

\begin{abstract}
Open source licenses provide everyone with the legal right to use, study, share, and improve the technology they cover from the perspective of copyright law. However, there are occasions when open source software packages or projects primarily governed by copyright licenses come into potential conflict with patent issues, or suffer from other governance concerns regarding third-party Intellectual Property Rights (IPR). From an economic perspective it is interesting how instead of undermining adoption, such challenges have led to an increase of collaborative governance solutions in open source, perhaps inspired by how such collaboration in development and business matters has provided benefit to stakeholders. In this paper, we show this evolution of collaborative solutions in open source business by actual example, and in the process illustrate how this unique approach to dealing with diverse ownership across business sectors works in practice.
\end{abstract}

\section{Categories and Subject Descriptors}

K.6.0 [Management of Computing and Information Systems]:

\section{General Terms}

Management, Economics, Experimentation, Legal Aspects

\section{Keywords}

Open Source License, Intellectual Property Rights, Collaboration, Governance, Open Invention Network.

\section{INTRODUCTION}

Business enterprises have always exercised their Intellectual Property Rights (IPR), especially around patent properties, in compliance with their business strategies. Takahashi (2007) indicates that the monetary value of patent properties primarily depends not on the scientific value per se but rather on the business assessment regarding its worth and the processes that frame such understanding. This means that aggression is possible with patents of low worth, but also that the opposite holds true. For example, even if a patent with value in terms of monopolizing a technology implementation is possessed by a business enterprise, the exertion of it is determined based on a strategic understanding of the requirements of the enterprise.

On the other hand, in the development process of Open Source, the sharing of ideas in a community of collaborators whatever form it takes, be it bazaar style or hierarchical - delivers more value than isolation in addressing challenges. Both are ultimately about strategic decisions about how to derive, maintain and grow value from the investment of resources, with the cooperative nature of the approaches in participation, licensing and patent use being based on a rational assessment of its utility. Moreover, with the latter generally being open to any participant, including those not associated with an organization, with the requisite knowledge of the software packages and the development style, while the former depends somewhat on the existence of business enterprises with IPR portfolios and investments.

Therefore, there occurs conflict between IPR portfolio decisions and Open Source business model. There are occasions when Open Source software packages or projects primarily governed by copyright licenses come into potential conflict with patent issues. Some Open Source licenses address this matter by the inclusion of patent provisions providing non-aggression pledges between collaborators on the licensed software, but the larger issue of whether a business makes a strategic decision to leverage patents aggressively essentially remains open. This is especially true of parties not collaborating on the same Open Source packages, or of third parties who may have minimal investment - and therefore understanding or sympathy - for Open Source approaches as a whole.

Noda, Tansho, and Coughlan (2012) describe this risk of conflicts and the possibility of collaborating solutions in Open Source business models. In this paper, we show this evolution of collaborative solutions in by actual example.

\section{CHECKS, BALANCES AND COOPERATION}

It is observable that in issues related to Open Source license compliance various checks and measures have been created to provide adherence to the rules and to discourage imbalances in the market. Examples include the lawsuits initiated by GPLviolations.org in Europe and later followed by Software Freedom Law Center in the USA, or the knowledge-sharing communities fostered by Linux Foundation and Free Software Foundation Europe to allow business leaders, developers and legal experts to both gain a greater understanding of issues faced and to formulate shared solutions where appropriate.

The most basic form of collaboration around Open Source can be seen in the development of Open Source code around the Linux kernel. There are numerous large and small stakeholders, ranging in size from multinational companies all the way down to individuals, and an even greater diversity of motivations for participation in the field. The energy and investment is loosely regulated via the mechanism of a central, independent body called 
the Linux Foundation, which acts as a sort of clearing house for ideas around the structure of new development and investment. By running conferences and meetings that allow stakeholders to interact, by hosting committees where industry standards regarding development are discussed and refined, and by hosting initiatives to realize the implementation of such standards, the Linux Foundation can be understood as a key example of collaboration around the governance of Open Source. The value it provides can be measured in dollar investment as well as less tangible expressions of support; the top tier of Linux Foundation corporate membership costs 500,000 USD per year.

Under this umbrella of general interaction more defined case studies may be found. From the perspective of business and legal affairs one key example is in the management of Open Source knowledge through the supply chain, an important step in both allowing companies to have confidence in receiving and further distributing Open Source, and a mechanism by which adherence to the legal obligations inherent to Open Source may be monitored. The Linux Foundation hosts a project called Software Package Data Exchange (SPDX), which is an emerging industry standard to apply a Bill of Materials (BoM) to software packages. The concept of a BoM comes from the hardware industry, and constitutes a formal description of all of the materials included in a package. By applying the same logic to software, and by ensuring that the industry forms a common approach around a single formal description for software materials, it becomes possible to greatly reduce the chance of costly business or legal errors when preparing products. Given that the modern supply chain to prepare a single product may contain multiple companies, in some cases even dozens, the need for such standardization is clear.

SPDX is not owned or operated by any single commercial entity. Instead it is created via a community of shared interest with a low barrier to entry, with various sub-committees chaired by volunteers assigned from companies investing substantial time in governance matters. In practice this means that the project discussions are hosted both physically and digitally by the Linux Foundation, with day to day administration of the discussions around the standard formation and further development being primarily addressed by commercial providers like Black Duck Software and OpenLogic who provide compatible tooling across the marketplace and in competition with each other. Contributors range from independent professionals involved in consultancy all the way through to representatives from very large companies acting as Tier 1 contractors in the supply chain. Each participant in the discussion has their own motives for contributing, and the evolution of the discussion is essentially governed or selfregulated by the combination of these motives. While the analogy may be imperfect, it is a microcosm of what would be termed a laissez-faire market for ideas when viewed from the perspective of economics. The application of supply and demand for practical results drives the evolution of the standard, and likewise ensures its utility for application in the real world.

\section{COLLABORATIVE GOVERNANCE}

Moving further into the area of legal risk, and more specifically the management of risk and obligations around Intellectual Property Rights (IPR), there is observable collaboration around both copyright and patent issues, perhaps the two most important segments of IPR to consider when discussing Open Source software. In the case of the former, and as alluded to in the introduction above, there is significant activity to share knowledge, expectations and best practices through channels provided by Linux Foundation and other, less narrowly-focused entities or groups. In the case of the Linux Foundation, there is an on-going project to hold "member counsel meetings" around major developer events, whereby legal representatives affiliated with companies and other entities working through the Linux Foundation have the opportunity to meet behind closed doors to review the current state of the market and to informally share their experiences and perceived requirements to facilitate the continued development and deployment of Open Source solutions. An example of a broadly focused group dealing with Open Source issues around copyright can likewise be found in the European Legal Network, a network facilitated by Free Software Foundation Europe, which allows over 280 legal experts from companies, NGOs and law firms to privately share knowledge and opinions both digitally via mailing lists and physically via a yearly meeting. These types of collaborative meetings and groups, while not entirely free for all, do allow key stakeholders to interact and to "raise the bar" on their mutual level of risk management. Their disadvantage is that they are inherently clubs of like-minded individuals or companies, and therefore do not directly "raise the bar" for the market as a whole. There are some socially constructed rules about who can be a member, and new entrants to the marketplace may face some barriers before they can participate.

In the case of patents and Open Source there have been slightly different solutions applied due to the different nature both of this form of IPR and the costs/risks inherent in its management. For example, while the costs associated with incorrect copyright decisions are generally limited to cease-and-desist notices, the possibility of individual products being delayed, and brand reputation damage, the costs associated with a single patent lawsuit run into millions of US dollars for defendants. Knowledge and opinion sharing is clearly not the key mechanism for engaging with such issues, and a more proactive stance regarding deterrent and defensive against common problems is required.

In the case of Open Source the key example of such an approach can be found in a specialized entity called Open Invention Network (OIN). While this holds the legal form of a company, it acts in a manner more akin - and complementary to neutral industry organizations like Linux Foundation. OIN was established in 2005 by Red Hat, IBM, NEC, Sony, Novell and Philips. Initially conceptualized as a shared pool of defensive patents and a common agreement not to litigate over a defined set of Linux System technologies, it now holds hundreds of defensive patents important to all sectors of technology, and has grown to lead a community of almost 500 companies and projects that formally pledge non-aggression to each other over the Linux System. It has also taken several steps to expand the technology included in its definition of the Linux System to match evolving market requirements, including broader coverage in the mobile and embedded markets. OIN currently represents the largest measure to mitigate patent risk in Open Source, and represents a fascinating balance between the application of patents as a temporary monopoly on a technology and the need for such monopolies to be waived over certain defined technology areas that inherently require unhindered and equal collaboration to maximize their value. In other words, it provides an example of how something like the Linux System can be conceptualized as a shared platform while also ensuring that outside of the shared 
platform - to use the language of software engineers, "higher in the stack" - the ability of each individual stakeholder to leverage their IPR for maximum benefit is unhindered.

Unpacking this concept a little more, while it makes sense for companies investing in the development of new technology to formally register such innovation in the form of patents, as previously discussed in this paper, what these companies subsequently seek to do with these patents is another matter. The aggressive enforcement of rights may provide initial advantage, but it can also reduce the ability of parties to collaborate in the mid to long-term, thus undermining the central precept behind obtaining lasting returns in Open Source. Taking this into account, the defensive holding of patents - and the strengthening of shared defensive pools and risk mitigation methods - is a logical business strategy. It is likely that OIN and perhaps similar entities will remain significant contributors to Open Source legal matters, and that the extent of its shared patent pool may increase as well. The reduction of potential risk from other collaborators on the Linux System in combination with the deterrent against aggression from third parties is a key motivator behind this rational decision.

\section{CONCLUSIONS}

From a broader perspective, it is possible to contextualize the modern governance activity around Open Source as being characterized by investors seeking to efficiently maintain the rules inherent to deriving value between diverse stakeholders, and to mitigate the risk of disruption from third parties wherever possible. This ensures the sustainability of value through collaboration, a state that facilitates a business environment with a wide range of applicable models and investors. This is manifested in collaborative approaches to code creation, platform management, and more specialized IPR issues. Whether such collaboration will further extend to nuanced solutions for shared supply chain management and other life-cycle management concerns is another matter. The more detailed and more specific a collaborative activity proposed, for example through the deferred use of patent monopolies, the more likely that some market participants may abstain from participation. This is especially true of activities that are not specific to Open Source per se, and which have an effect or are conversely influenced by more general market concerns. In the case of patent non-aggression, important third parties may include software companies who not only do not see value in protecting an Open Source technology like the Linux System, but who actively would wish to compete against it. For these reasons the intersection between IPR portfolio decisions and Open Source business imperatives, and tension between commercial and collaborative life-cycle knowledge provision may be an interesting vein of potential further study, especially when considered in conjunction with the multi-layered relations between Open Source and proprietary software companies.

\section{REFERENCES}

[1] Andrew, M. 2004. Understanding Open Source \& Free Software Licensing. O’Reilly Publishing.

[2] Guadamuz, A. 2004. Viral Contracts or Unenforceable Documents? Contractual Validity of Copyleft Licenses. E.I.P.R 26(8), 331-339.

[3] Hatta, M. 2007. Software License and the Development Style. In Licensing Strategy, Takahashi, N. and Nakano, K. Ed. Manufacturing Management Research Center of Tokyo University, 197-238.

[4] Noda, T., Tansho. T., and Coughlan, S. 2012. The Effect of Open Source Licensing on the Evolution of Business Strategy. In Proceedings of 8th IFIP WG 2.13 International Conference, on Open Source Systems. Hammouda, I., Lundell, B., Mikkonen, T., and Scacchi. W. Ed. Springer. 344-349.

[5] Noda, T., Tansho. T., and Cai, L. 2012. Development Style of Open Source Software and Innovation of Business Enterprises International. In International Proceedings of Economics Development and Research Volume 39, 16-20.

[6] Noda, T., Cai, L., and Coughlan, S. 2012. Open Source License and Transition of Governance in Business Enterprises. In International Proceedings of Economics Development and Research Volume 57, 13-17.

[7] Takahashi, N. 2007. General Theory of the Strategy of Licensing Business, Software License and Development Style. In Licensing Strategy, Takahashi, N. and Nakano, K. Ed. Manufacturing Management Research Center of Tokyo University, 47-94.

[8] Wong, C., Kreps, J. 2009. Collaborative Approach: Peer-toPatent and the open source movement. In International Free and Open Source Software Law Review. Information, Volume 01 Issue 01, 15-26 